\section{ORIGINAL RESEARCH}

\author{
N.D. Ha \\ Y.C. Weon \\ J.C. Jang \\ B.S. Kang \\ S.H. Choi
}

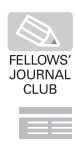

\title{
Spectrum of MR Imaging Findings in Wernicke Encephalopathy: Are Atypical Areas of Involvement Only Present in Nonalcoholic Patients?
}

BACKGROUND AND PURPOSE: Although MR imaging is considered the most effective method to confirm a diagnosis of WE, MR imaging studies designed to distinguish WE between NA and AL patients have yielded controversial results. The purpose of this study was to determine potential differences in MR imaging features between AL and NA patients with WE and to compare neurologic symptoms with MR imaging findings.

MATERIALS AND METHODS: This retrospective study included 24 consecutive patients (male/female, 15:9; mean age, 54 years) diagnosed with $W E$ in a university hospital ( $A L=13, N A=11$ ). Clinical manifestations and MR imaging findings between AL and NA patients were evaluated. Classic WE symptom triad and consciousness level and MR imaging findings were scored and compared with each other. Statistical analyses were performed with $\chi^{2}$, Fisher exact, and Spearman tests.

RESULTS: No differences were observed regarding the areas of hyperintense signal intensity on FLAIR imaging and enhancement of the mammillary bodies between AL and NA patients $(P>.05)$. Frequent sites of involvement were the medial thalami $(86 \%)$, dorsal medulla $(82 \%)$, tectal plate $(77 \%)$, and the periaqueductal gray matter $(75 \%)$. A positive association was found between the consciousness levels of the patients and the involvement of atypical sites $(P=.01)$. Only 4 of the 24 patients $(17 \%)$ had all 3 symptoms of the classic WE symptom triad.

CONCLUSIONS: MR imaging features of WE may not be different between AL and NA patients. The medulla is 1 of the most frequently involved sites, and consciousness level is also associated with atypical site involvement.

ABBREVIATIONS: $A L=$ alcoholic; $N A=$ nonalcoholic; NIAAA = National Institute on Alcohol Abuse and Alcoholism; SENSE = sensitivity encoding; WE = Wernicke encephalopathy

$\mathbf{W}$ $E$ is a life-threatening emergent neurologic disorder resulting from thiamine (vitamin $B_{1}$ ) deficiency, and its prognosis depends on prompt early diagnosis followed by intravenous administration of thiamine. ${ }^{1-3} \mathrm{WE}$ is clinically characterized by mental status changes, ocular symptoms, and motor problems, such as gait incoordination and ataxia. WE is commonly associated with prolonged alcohol abuse and is more readily diagnosed in AL patients, while WE in NA patients may be prone to misdiagnosis or a delay in diagnosis. ${ }^{2,4-7}$ Currently, the most effective method to confirm a diagnosis of WE in both NA and AL patients is MR imaging.

Typical MR imaging of WE usually shows symmetric T2 signal intensity alterations in the medial thalami, mammillary bodies, tectal plate, and periaqueductal area, ${ }^{8}$ whereas signalintensity alterations in the cerebellum, including the vermis, cranial nerve nuclei, red nuclei, dentate nuclei, caudate nuclei, splenium, and cerebral cortex, represent atypical MR imaging findings. ${ }^{4,69-20}$ Recently, some studies have been able to differentiate NA patients from AL patients according to differ-

Received September 22, 2011; accepted after revision November 11.

From the Department of Radiology, Ulsan University Hospital, University of Ulsan College of Medicine, Ulsan, Republic of Korea.

Please address correspondence to Young Cheol Weon, MD, Department of Radiology, Ulsan University Hospital, 290-3 Jeonha-Dong, Dong-Gu, Ulsan 682-714, Republic of Korea; e-mail: ycweon@hanmail.net

Indicates article with supplemental on-line table.

http://dx.doi.org/10.3174/ajnr.A2979 ences in MR imaging findings. Zuccoli et $\mathrm{al}^{8,21}$ suggested that $\mathrm{AL}$ and NA patients present different MR imaging patterns due to differences in the pathophysiologic processes. In these studies, atypical MR imaging findings, such as signal-intensity alterations of the cerebral cortex and lower cranial nerve nuclei, were only observed in NA patients. ${ }^{8,22}$ In contrast, other studies have reported several cases of AL patients with WE who also showed atypical brain MR imaging findings. ${ }^{23,24}$ These findings suggest that further investigations are required before the relationship between alcohol and the brain lesions associated with WE can be understood. In our daily clinical practice, we have observed atypical brain MR imaging findings, not only in NA patients but also in AL patients who presented with WE. This retrospective study was performed to examine any potential differences in MR imaging findings between $\mathrm{AL}$ and NA patients and to compare the neurologic symptoms, including consciousness levels, with MR imaging findings.

\section{Materials and Methods}

\section{Patients}

This retrospective study included 24 consecutive patients (15 men and 9 women; mean age, 54 years) diagnosed with WE between April 2004 and November 2010 in a university hospital. Diagnosis of WE was made on the basis of the criteria of Caine et al, ${ }^{24}$ which require 2 of the following 4 items: dietary deficiencies, ocular symptoms, ataxia, 
and an altered consciousness or memory impairment. We reviewed the patient medical records, onset date, patient treatment history, underlying diseases, causes of death, and neurologic symptoms, such as altered consciousness, ocular symptoms, ataxia, and so forth. A detailed history of alcohol intake and habits was taken to distinguish chronic alcoholics on the basis of the diagnostic criteria of the NIAAA. All patients were of Korean descent. This retrospective study was approved by the institutional review board of our hospital.

\section{Clinical Assessment}

Consciousness scores were determined according to the level of consciousness at presentation based on neurologic examination: 1 , alert; 2, drowsy; 3 , stupor; 4 , semicoma; 5 , coma. Symptom scores were calculated by adding 1 point when the patient had 1 of the symptoms of the WE triad: altered consciousness, ocular symptoms, or ataxia.

\section{MR Imaging Technique}

MR imaging was performed on either a 3T system (Intera Achieva; Philips Healthcare, Best, the Netherlands) or a 1.5T system (Achieva; Philips Healthcare) by using an 8-channel SENSE head coil or a 16channel SENSE Neurovascular Coil (Invivo, Gainesville, Florida). The standard protocol for WE included T1WI, T2WI, FLAIR, contrast-enhanced T1WI, and DWI with ADC maps. The FLAIR image was obtained with a $352 \times 256$ matrix (TR/TE/TI/NEX, 11,000 ms/ $125 \mathrm{~ms} / 2800 \mathrm{~ms} / 2)$ at $3 \mathrm{~T}$ and a $256 \times 251$ matrix (TR/TE/TI/NEX, $6000 \mathrm{~ms} / 120 \mathrm{~ms} / 2000 \mathrm{~ms} / 2$ ) at $1.5 \mathrm{~T}$. The sequence parameters for T1WI were a $244 \times 256$ matrix (TR/TE/NEX, $560 \mathrm{~ms} / 15 \mathrm{~ms} / 2$ or 1$)$ at $3 \mathrm{~T}$ and a $256 \times 230$ matrix (TR/TE/NEX, $450 \mathrm{~ms} / 12 \mathrm{~ms} / 2)$ at $1.5 \mathrm{~T}$. The sequence parameters for T2WI were a $400 \times 320$ matrix (TR/TE/ NEX, $3000 \mathrm{~ms} / 80 \mathrm{~ms} / 1)$ at $3 \mathrm{~T}$ and a $320 \times 320$ matrix $(\mathrm{TR} / \mathrm{TE} / \mathrm{NEX}$, $4000 \mathrm{~ms} / 100 \mathrm{~ms} / 2$ ) at $1.5 \mathrm{~T}$. The section thickness of FLAIR, T1WI, and T2WI was $5 \mathrm{~mm}$ with a 2-mm intersection gap for each sequence. Gadopentetate dimeglumine (Magnevist; Schering, Berlin, Germany) was administered at a dose of $0.1 \mathrm{mmol} / \mathrm{kg}$ of body weight. Axial FLAIR images were obtained in all patients. Enhanced T1WI (axial and sagittal) and axial ADC maps were available in 18 and 14 patients, respectively.

\section{MR Imaging Assessment}

Three radiologists - 1 neuroradiologist, 1 pediatric radiologist (including pediatric neuroradiology), and 1 musculoskeletal radiologist (10, 8 , and 8 years' experience, respectively)—retrospectively reviewed the brain MR images, blinded to the clinical symptoms or the etiology of WE, and a consensus was reached in conference. MR imaging results of WE were considered typical if symmetric hyperintense signal intensities were observed in the medial thalami, mammillary bodies, periaqueductal gray matter, periventricular region of the third and fourth ventricles, and the tectal plate on FLAIR images. MR imaging results of WE were considered atypical when signal-intensity alterations were observed in the cerebellum, cerebral cortex, medulla oblongata, corpus callosum, cranial nerve nuclei, and basal ganglia on FLAIR images. Typical and atypical MR imaging findings were compared between AL and NA groups, and imaging scores were calculated by adding 1 point according to the number of sites involved on the FLAIR images. Imaging scores were also compared with patient clinical symptoms, including the consciousness score. We also assessed enhancing lesions on contrast-enhanced T1WI in the AL and NA groups.

\section{Statistical Analyses}

The statistical significance of the association between alcohol abuse and brain MR imaging findings was calculated with $\chi^{2}$ and Fisher exact tests. The significance of the association between alcohol abuse and symptom score and between consciousness level (or symptom score) and brain MR imaging findings was analyzed by the $\chi^{2}$ test. The correlation between consciousness level (or symptom score) and imaging score (typical and atypical) was calculated with the Spearman test. The prevalence of the WE symptom triad between AL and NA groups was also compared. Independent factors were expressed as relative risk with a $95 \%$ confidence interval. All $P$ values $<.05$ were considered to indicate statistical significance. The statistical analyses were performed by using a software package (Statistical Package for the Social Sciences, Version 17.0; SPSS, Chicago, Illinois).

\section{Results}

\section{Clinical Data}

The On-line Table summarizes the clinical data of the 24 patients included in this study. Eleven of the 24 patients (46\%) with WE fulfilled the diagnostic criteria of alcoholism according to the NIAAA. The alcoholic patients consumed an average of $21 \%$ alcohol (approximately $767.7 \pm 407 \mathrm{~mL}$ ) per day over $11 \pm 6.8$ years. The consumed ethanol was mainly in the form of distilled liquor.

The remaining 13 patients (54\%) were NA. The most frequent cause of thiamine deficiency in the NA group was malnutrition secondary to malignancy or another medical condition (10/13 NA patients). Three of the 13 NA patients had malnutrition due to anorexia nervosa, poor oral intake due to diet, and poor oral intake associated with SAH, respectively.

\section{Neurologic Features}

The On-line Table describes the neurologic symptoms of study patients and their calculated symptom and consciousness scores. Eleven of 24 patients showed alterations of consciousness (46\%, 4 AL/7 NA). Among these 11 patients, 7 were drowsy (29\%, 3 AL/4 NA), 1 showed stupor (4\%,1 NA), 2 were semicomatose $(8 \%, 1 \mathrm{AL} / 1 \mathrm{NA})$, and 1 was in a coma $(4 \%, 1$ NA). Seventeen of the 24 patients were evaluated as having a neurologic deficit. Ten of these 17 patients showed ocular symptoms, including nystagmus, diplopia, and ophthalmoplegia (59\%, $7 \mathrm{AL} / 3 \mathrm{NA}$ ), and 9 of the 17 showed ataxia (53\%, 6 AL/3 NA). However, ocular symptoms and ataxia did not differ statistically between AL and NA groups with WE $(P=$ .362 and .350 , respectively). Other reported symptoms included vertigo, amnesia, headache, and dizziness.

\section{Neuroradiologic Features}

Two of the 24 patients were excluded from MR imaging analysis due to normal brain MR imaging findings for 1 patient and the lack of MR imaging for the other patient. The mean time interval between the onset of symptoms and the first MR imaging study of 21 patients was 4.5 days with the exception of 1 patient (44 days). The time interval was longer in the NA group (mean, 4 days) than in the AL group (mean, 2 days).

The Table shows the areas of hyperintense signal intensity on FLAIR imaging in 22 patients. The sites showing involvement on the FLAIR images were the periaqueductal gray matter in $21 / 22(95 \%)$, the medial thalami in $19 / 22(86 \%)$, the 


\begin{tabular}{|c|c|c|c|c|c|c|c|c|c|c|c|c|}
\hline \multicolumn{13}{|c|}{ Topographic distribution of the lesion ${ }^{\mathrm{a}}$ in AL and NA patients with WE } \\
\hline $\begin{array}{l}\text { Patient } \\
\text { Group }\end{array}$ & PeriG & $\mathrm{T}$ & Third & Medulla & Tectum & MB & Fourth & Cortex & CN9 & Cbll & BG & Corpus \\
\hline$\overline{\mathrm{AL}}$ & 9 & 7 & 8 & 6 & 6 & 5 & 4 & 3 & 1 & 1 & 0 & 1 \\
\hline NA & 12 & 12 & 11 & 12 & 11 & 7 & 2 & 2 & 4 & 2 & 1 & 0 \\
\hline Total (\%) & $21(95)$ & $19(86)$ & $19(86)$ & 18 (82) & 17 (77) & $12(55)$ & $6(27)$ & $5(23)$ & $5(23)$ & $3(14)$ & $1(5)$ & $1(5)$ \\
\hline
\end{tabular}

Note:-PeriG, periaqueductal gray matter; T, medial thalami; Third, periventricular region of the third ventricle; Medulla, dorsal medulla; Tectum, tectal plate; MB, mamillary bodies; Fourth periventricular gray matter of the fourth ventricle; Cortex, cerebral cortex; CN9, ninth cranial nuclei; Cbll, cerebellum, BG, basal ganglia; Corpus, splenium of corpus callosum. aigh-signal-intensity regions on FLAIR imaging.
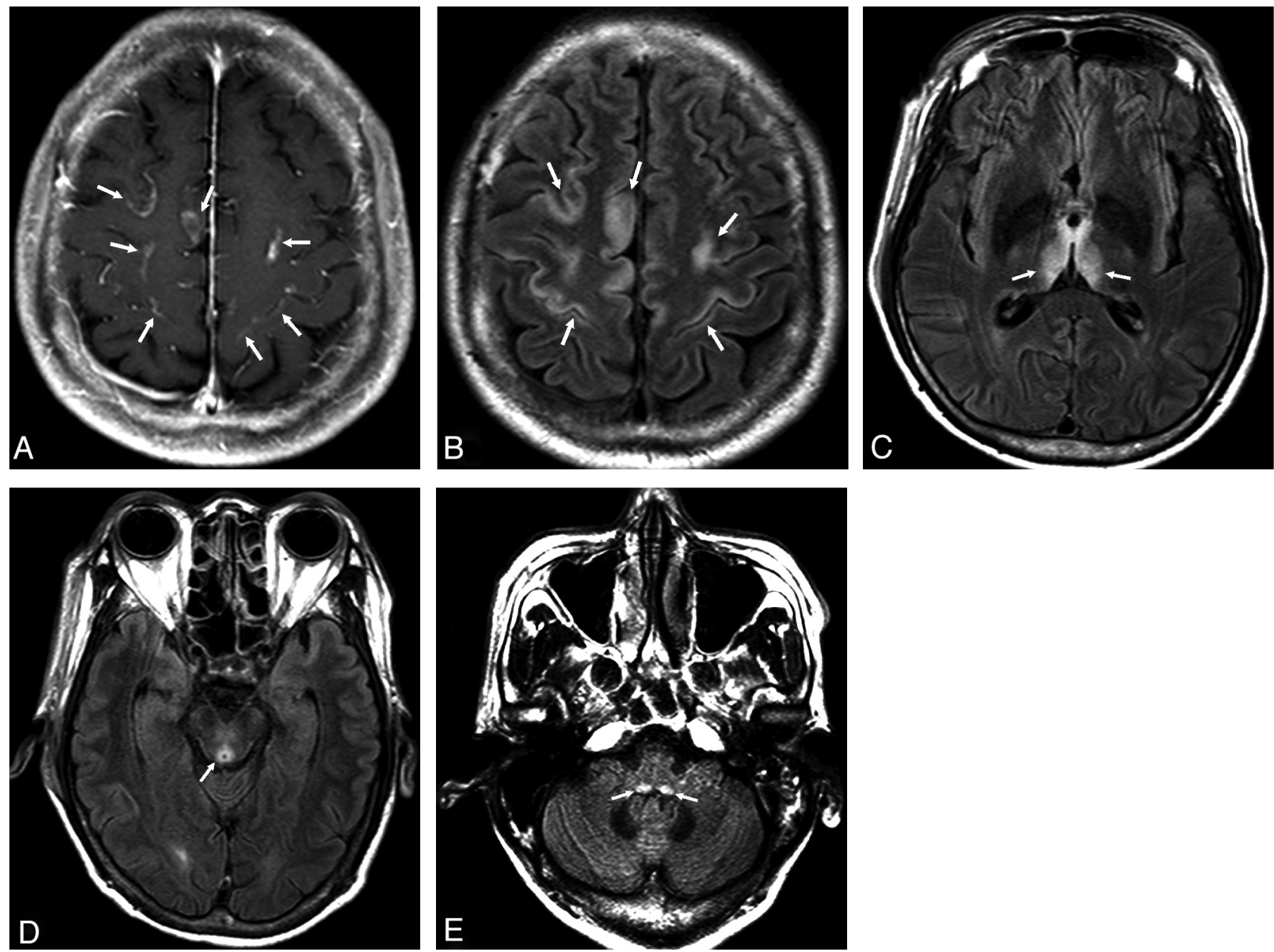

Fig 1. A 57-year-old man with a history of alcohol abuse presented with altered consciousness, ocular symptoms, and ataxia. $A$, Contrast-enhanced T1WI shows contrast enhancement in the cortical area of the frontal and parietal lobes (arrows). B-E, FLAIR axial images. B, Diffuse asymmetric signal-intensity alterations in the cortical area of the frontal and parietal lobes (arrows). C, Signal-intensity alterations in the medial thalami (arrows). D, Signal-intensity alteration in the periaqueductal gray matter (arrow). E, Signal-intensity alterations of medulla oblongata (arrows).

periventricular region of the third ventricle in $19 / 22(86 \%)$, the dorsal medulla in $18 / 22(82 \%)$, the tectal plate in $17 / 22$ (77\%), the mammillary bodies in $12 / 22$ (55\%), the periventricular gray matter of the fourth ventricle in $6 / 22$ (27\%), the cerebral cortex in 5/22 (23\%), the ninth cranial nerve nuclei in $5 / 22(23 \%)$, the cerebellum in $3 / 22(14 \%)$, the splenium of corpus callosum in $1 / 22(5 \%)$, and the basal ganglia in $1 / 22$ (5\%). No significant differences between AL and NA patients were seen in the areas of involvement on FLAIR images $(P>$ $.05)$. In addition, no significant differences in imaging scores between AL and NA groups were revealed. Furthermore, alcohol abuse had no statistically significant association with signal-intensity alteration of the cerebral cortex on brain MR imaging in our study $(P=.618)$. Among the patients with involvement of the cerebral cortex (AL 3/NA 2), 3 (AL 1/NA 2) showed asymmetric signal-intensity alterations in the cortical area of the frontal and parietal lobes (Fig 1). Two other AL patients also showed asymmetric signal-intensity alterations in the cortical area of the frontal lobe.

Contrast-enhanced T1WI was performed in 17 of 22 patients. On contrast-enhanced MR images, no differences in enhancement sites between the AL and NA groups were found. Enhancements were present in the tectum in $12 / 17$ (71\%, AL 4/NA 8), periaqueductal gray matter in $7 / 17$ (41\%, AL 3/NA 4), medial thalami in 6/17 (35\%, AL 3/NA 3), mammillary bodies in $6 / 17$ (35\%, AL 2/NA 4), periventricular region of the third ventricle in 5/17 (29\%, AL 3/NA 2), dorsal medulla in $3 / 17$ (18\%, AL 1/NA 2), periventricular gray matter 
of the fourth ventricle in $2 / 17(12 \%, \mathrm{AL} 1 / \mathrm{NA} 1)$, cerebral cortex in $2 / 17(12 \%, 2 \mathrm{AL})$, and ninth cranial nerve nuclei in $1 / 17$ (6\%, NA 1).

\section{Neurologic Symptoms and MR Imaging Features}

Symptom scores based on the classic symptom triad of WE were calculated in 17 of 24 patients (AL 10/NA 7). The symptom scores of patients with WE were not statistically different between the AL and NA groups $(P=.1)$. The symptom scores of patients with WE were also not statistically associated with the calculated imaging score; however, this finding may be due to the fact that mental status changes in some patients made it impossible to evaluate the other symptoms, particularly ataxia. In addition, the consciousness level of the patients was not always associated with the total extent of the involved sites (total imaging score) on FLAIR images. The consciousness level, however, had a positive correlation with involvement of the atypical areas (atypical imaging score, $R=.623, P=.01$ ) of the brain on MR imaging. Among these, cerebral cortex involvement was significantly associated with consciousness level $(P=.006)$ and symptom score $(P=.007)$, respectively.

\section{Discussion}

\section{Brain MR Imaging Findings of WE in AL and NA Patients}

Our results suggest that MR imaging findings may not differ between AL and NA patients. The atypical MR imaging findings, including cerebral cortex and cranial nerve nuclei lesions, were present in the AL group and the NA group in our study. Contrast enhancement in the mammillary bodies and medial thalami, a typical finding in the AL group, was also present in the NA group. Thus, alcohol abuse had no statistically significant positive association with any involved brain area on MR imaging in patients with WE in our study. However, previous studies, such as the one by Zuccoli et al, ${ }^{21}$ who studied 56 patients with WE, have suggested that brain images of NA patients with WE may differ from those of AL patients with WE. These authors reported that signal-intensity alterations in the areas considered atypical for WE were noted only in the NA patients and that contrast enhancement of the medial thalami and mammillary bodies is a typical finding of WE in AL patients, but not in NA patients. ${ }^{8,22}$ They further suggested that because atypical lesions are only present in WE in NA patients, alcohol may have a protective effect on the brain, and as a consequence, MR imaging can help distinguish AL from NA patients with WE. ${ }^{25}$ Some reports, however, have shown that atypical MR imaging findings are present in AL patients with WE. ${ }^{22,23}$ These reports further showed signal-intensity alterations of the cerebral cortex and amygdala in $2 \mathrm{AL}$ patients. Our study, in agreement with these latter reports, did not find any significant association between alcohol abuse and MR imaging findings in WE. Therefore, we speculated that WE pathophysiology and MR imaging findings might not differ between AL and NA patients because WE is caused by a thiamine deficiency irrelevant to alcohol abuse.

\section{Typical and Atypical MR Imaging Findings of WE}

In this study, brain MR imaging findings usually showed symmetric signal-intensity alterations in the medial thalami
$(86 \%)$, periaqueductal gray matter $(75 \%)$, periventricular region of the third ventricle (73\%), tectal plate (77\%), and dorsal medulla (82\%). These MR imaging results confirm that the regions most frequently involved in WE are the medial thalami and the periventricular region of the third ventricle. ${ }^{8}$ These areas are considered typical sites involved in WE, and these findings may be explained by the maintenance of cellular osmotic gradients that are strictly related to the concentration of thiamine levels in these areas. ${ }^{26}$ Although previous studies have not mentioned it, the dorsal medulla was 1 of the most frequently involved regions in the present study. The medulla oblongata contains many cranial nerve nuclei, but these cranial nerve nuclei cannot be distinguished on the FLAIR images in our study. Therefore, we describe signal-intensity changes of the dorsal medulla rather than signal-intensity changes of each lower cranial nerve nuclei. Signal-intensity alterations of atypical locations, including the cerebral cortex (23\%), ninth cranial nerve nuclei (23\%), corpus callosum (5\%), basal ganglia $(5 \%)$, and cerebellum (14\%), are similar to those in previous reports. ${ }^{4,6,8-20}$

\section{Consciousness Status in Atypical WE MR Imaging Findings}

In this study, consciousness scores have a statistically positive correlation with the atypical sites involved and have significant association with the involvement of the cerebral cortex. These results could indicate that the more severe the consciousness change, the more atypical the MR imaging findings. Approximately $82 \%$ of patients have mental status changes according to an autopsy-based series. ${ }^{27}$ These changes largely result from an involvement of the medial thalami or mammillary bodies and range from a confused state to mental sluggishness, apathy, impaired awareness of the immediate situation, inability to concentrate, and, if left untreated, coma and death. Fei et $\mathrm{al}^{13}$ reported that the presence of cortical lesions in association with coma may indicate a poor prognosis as a consequence of irreversible brain damage. Our 5 patients (3 AL/2 NA) with cerebral cortex involvement in brain MR imaging also presented with a poor prognosis. Therefore, we hypothesize that the severity of symptoms and prognosis may be related to the involvement of the medial thalami and mammillary bodies as well as to atypical sites of involvement, especially the cerebral cortex in WE.

\section{Classic Symptom Triad of WE}

The clinical diagnosis of WE, which has traditionally rested on the presence of the classic symptom triad (altered consciousness, ocular symptoms, and ataxia), is not always easy because the classic symptom triad is found in only $16 \%-38 \%$ of all patients with WE. ${ }^{8,27}$ Moreover, approximately $19 \%$ of patients have none of the symptoms of the classic triad at presentation with WE. ${ }^{27}$ In our study, only 4 of 24 patients (17\%) had all 3 of the classic symptoms. Altered consciousness levels were shown in 11 of 24 patients (46\%) and were higher in the NA group than in the AL group (7/13 and 4/11, respectively). In contrast, ocular symptoms and ataxia, in patients in whom these symptoms could be evaluated, were more frequent in the AL group than in the NA group. Ocular symptoms were present in 8/11 AL patients and in 3/8 NA patients, while ataxia was present in 7/10 AL patients and 3/7 NA patients. These results 
are in agreement with those in a previous report. ${ }^{24}$ The prevalence of ocular symptoms and ataxia, however, did not differ statistically between AL and NA groups in this study. Because the WE symptom triad could appear less frequently in the NA group, this group may present more of a challenge in reaching an early diagnosis in the clinical setting than the AL group; these latter patients are usually admitted to the emergency department with a history of alcoholism. The fact that the time interval between symptom onset and first the MR imaging study was longer in the NA group (mean, 4 days) than in the AL group (mean, 2 days, except for 1 patient with a 44-day interval) also supports the idea that the NA group is more difficult to diagnose than the AL group. Thus, MR imaging could be a powerful tool in supporting the diagnosis of WE in $\mathrm{AL}$ and NA patients and could also help distinguish WE from other emergent neurologic disorders, especially in patients with mental status changes.

\section{Limitations}

Although the present study provides significant insight into MR imaging as a diagnostic tool for WE in AL and NA patients, our study also has certain limitations that should be considered when interpreting the results. These limitations include the relatively small number of patients in this study and its retrospective nature; however, the patients included were consecutively collected in a university hospital during more than a 6-year period so that MR images were obtained from the same units, $1.5 \mathrm{~T}$ or $3 \mathrm{~T}$, by using consistent imaging protocols. It is possible that WE could be underdiagnosed clinically, leading to some degree of selection bias. Additionally, an underestimation of the involvement of mammillary bodies and the floor of the fourth ventricle is possible due to the relatively thick sections and section intervals in axial images used in this study. Moreover, it was not possible to obtain all coronal and sagittal FLAIR images for patients. However, the symmetric alterations in the mammillary bodies were evident in $55 \%$ of our patients, and this prevalence is not different from that in previous reports. Additional, more comprehensive studies involving a larger number of patients will be necessary to confirm these results.

\section{Conclusions}

MR imaging findings of WE may not differ between AL and NA patients. Atypical MR imaging findings of WE, including cerebral cortical lesions and cranial nerve nuclei lesions, were present in the AL group as well as the NA group. Contrast enhancement in the mammillary bodies and medial thalami was also present in both the AL and NA groups. The most common sites involved in WE were the periaqueductal gray matter, medial thalami, tectum, and periventricular region of third ventricle, with the dorsal medulla being 1 of the most frequently involved sites. Consciousness may be related to involvement of the medial thalami and mammillary bodies as well as involvement of the atypical sites, including the cerebral cortex. Although MR imaging cannot differentiate NA from AL patients, it could still be a helpful tool in supporting the diagnosis of WE in both the AL and NA groups and could also help distinguish WE from other emergent neurologic disorders, especially in patients with mental status changes.

\section{References}

1. Harper C, Rodriguez M, Gold J, et al. The Wernicke-Korsakoff syndrome in Sydney: a prospective necropsy study. Med J Aust 1988;149:718-20

2. Ogershok PR, Rahman A, Nestor S, et al. Wernicke encephalopathy in nonalcoholic patients. Am J Med Sci 2002;323:107-11

3. Reuler JB, Girard DE, Cooney TG. Current concepts: Wernicke's encephalopathy. N Engl J Med 1985;312:1035-39

4. Zhong C, Jin L, Fei G. MR imaging of nonalcoholic Wernicke encephalopathy: a follow-up study. AJNR Am J Neuroradiol 2005;26:2301-05

5. Sun GH, Yang YS, Liu QS, et al. Pancreatic encephalopathy and Wernicke encephalopathy in association with acute pancreatitis: a clinical study. World J Gastroenterol 2006;12:4224-27

6. Liu YT, Fuh JL, Lirng JF, et al. Correlation of magnetic resonance images with neuropathology in acute Wernicke's encephalopathy. Clin Neurol Neurosurg 2006;108:682-87

7. Gui QP, Zhao WQ, Wang LN. Wernicke's encephalopathy in nonalcoholic patients: clinical and pathologic features of three cases and literature reviewed. Neuropathology 2006;26:231-35

8. Zuccoli G, Gallucci M, Capellades J, et al. Wernicke encephalopathy: MR findings at clinical presentation in twenty-six alcoholic and nonalcoholic patients. AJNR Am J Neuroradiol 2007;28:1328-31

9. Bae SJ, Lee HK, Lee JH, et al. Wernicke's encephalopathy: atypical manifestation at MR imaging. AJNR Am J Neuroradiol 2001;22:1480-82

10. Bonucchi J, Hassan I, Policeni B, et al. Thyrotoxicosis associated Wernicke's encephalopathy. J Gen Intern Med 2008;23:106-09

11. D'Aprile P, Tarantino A, Santoro N, et al. Wernicke's encephalopathy induced by total parenteral nutrition in patient with acute leukaemia: unusual involvement of caudate nuclei and cerebral cortex on MRI. Neuroradiology 2000;42:781-83

12. Doss A, Mahad D, Romanowski CA. Wernicke encephalopathy: unusual findings in nonalcoholic patients. J Comput Assist Tomogr 2003;27:235-40

13. Fei GQ, Zhong C, Jin L, et al. Clinical characteristics and MR imaging features of nonalcoholic Wernicke encephalopathy. AJNR Am J Neuroradiol 2008; 29:164-69

14. Kang SY, Kang JH, Choi JC, et al. Wernicke's encephalopathy: unusual manifestation on MRI. J Neurol 2005;252:1550-52

15. Kim HA, Lee H. Atypical Wernicke's encephalopathy with remarkable cerebellar lesions on diffusion-weighted MRI. Eur Neurol 2007;58:51-53

16. Lapergue B, Klein I, Olivot JM, et al. Diffusion weighted imaging of cerebellar lesions in Wernicke's encephalopathy. J Neuroradiol 2006;33:126-28

17. Loh Y, Watson WD, Verma A, et al. Restricted diffusion of the splenium in acute Wernicke's encephalopathy. J Neuroimaging 2005;15:373-75

18. Murata T, Fujito T, Kimura H, et al. Serial MRI and (1)H-MRS of Wernicke's encephalopathy: report of a case with remarkable cerebellar lesions on MRI. Psychiatry Res 2001;108:49-55

19. Nolli M, Barbieri A, Pinna C, et al. Wernicke's encephalopathy in a malnourished surgical patient: clinical features and magnetic resonance imaging. Acta Anaesthesiol Scand 2005;49:1566-70

20. Zuccoli G, Motti L. Atypical Wernicke's encephalopathy showing lesions in the cranial nerve nuclei and cerebellum. J Neuroimaging 2008;18:194-97

21. Zuccoli G, Santa Cruz D, Bertolini M, et al. MR imaging findings in 56 patients with Wernicke encephalopathy: nonalcoholics may differ from alcoholics. AJNR Am J Neuroradiol 2009;30:171-76

22. Hygino da Cruz LC Jr, Domingues RC, Vilanova I, et al. MR imaging findings in Wernicke encephalopathy: nonalcoholics may be similar to alcoholics. AJNR Am J Neuroradiol 2010;31:E54-55, author reply E6

23. Sugai A, Kikugawa K. Atypical MRI findings of Wernicke encephalopathy in alcoholic patients. AJR Am J Roentgenol 2010;195:W372-73, author reply W4

24. Caine D, Halliday GM, Kril JJ, et al. Operational criteria for the classification of chronic alcoholics: identification of Wernicke's encephalopathy. J Neurol Neurosurg Psychiatry 1997;62:51-60

25. Zuccoli G, Pipitone N. Neuroimaging findings in acute Wernicke's encephalopathy: review of the literature. AJR Am J Roentgenol 2009;192: 501-08

26. Zuccoli G, Siddiqui N, Bailey A, et al. Neuroimaging findings in pediatric Wernicke encephalopathy: a review. Neuroradiology 2010;52:523-29

27. Harper CG, Giles M, Finlay-Jones R. Clinical signs in the Wernicke-Korsakoff complex: a retrospective analysis of 131 cases diagnosed at necropsy. J Neurol Neurosurg Psychiatry 1986;49:341-45 